\title{
Modelling of the Coherent Backscattering Opposition Effect: Comparison to Planetary Phase Curves
}

\author{
Sanna Kaasalainen, Karri Muinonen, Jukka Piironen \\ Observatory, P.O. Box 14, FIN-00014 University of Helsinki, Finland
}

\begin{abstract}
We study the opposition effect of icy planetary satellites by means of a Monte Carlo simulation of the coherent backscattering opposition effect for spherical objects covered with locally plane parallel media.
\end{abstract}

The phase curve of Europa's leading face (Thompson \& Lockwood 1992), showing a relatively sharp opposition peak, is interpreted with the aid of an exponential-linear fitting function

$$
I(\alpha)=I_{s} \exp \left(-\frac{\alpha}{2 \ell}\right)+I_{b}+k \alpha,
$$

where $I_{s}$ is the size and $\ell$ the width of the opposition peak, $I_{b}$ is the background part, and $k$ the slope of the linear part. To evaluate the goodness of the fit, the marginal probability distribution for the width and size of the opposition peak (Karttunen \& Muinonen 1991) is computed (Fig. 1). If there are enough accurate data points at the small phase angle region, a clear and narrow maximum occurs, corresponding to the best fit of the parameters, whereas a wide distribution would imply too high imprecision in the data. Coherent backscattering is fitted indirectly to the data by combining the width $\ell$ from the fit of Eq. (1) to the simulated phase curve, and the linear parameters from the fit to the observations (Fig. 1).
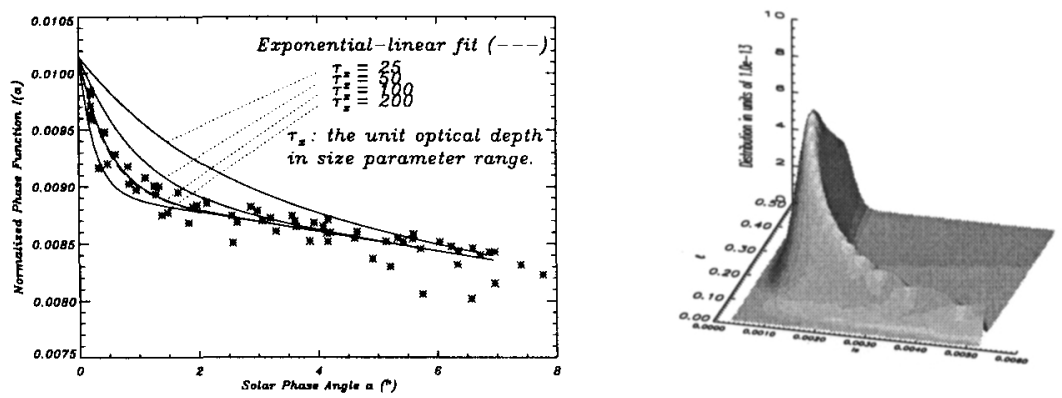

Figure 1. Europa's phase curve and the marginal probability density.

\section{References}

Karttunen, H., \& Muinonen, K. 1991, A\&A, 242, 513

Thompson, D. T., \& Lockwood, G. W. 1992, JGR, 97, 14761 